\title{
La incursión del Congreso Nacional de Cirugía de Colombia en la red social Twitter ${ }^{\mathrm{TM}}$
}

\author{
The Colombian National Congress of Surgery's Breakthrough into \\ the Social Network Twitter
Diego Sierra1', Elio Fabio Sánchez², Sebastián Sierra³ ${ }^{3}$ Alfonso Carlos Márquez², Lilian Torregrosa ${ }^{4}$

1 Médico cirujano; profesor, Departamento de Cirugía, Facultad de Medicina, Universidad de La Sabana, Chía; miembro, Comité de Comunicaciones, Asociación Colombiana de Cirugía, Bogotá, D.C., Colombia

2 Médico cirujano; profesor, Departamento de Cirugía, Facultad de Medicina, Pontificia Universidad Javeriana; miembro, Comité de Comunicaciones, Asociación Colombiana de Cirugía, Bogotá, D.C., Colombia

3 Médico cirujano; profesor, Departamento de Cirugía, Facultad de Medicina, Universidad de Antioquia, Medellín; miembro, Comité de Comunicaciones, Asociación Colombiana de Cirugía, Bogotá, D.C., Colombia

4 Médica cirujana; directora, Departamento de Cirugía, Facultad de Medicina, Pontificia Universidad Javeriana; vicepresidente, Asociación Colombiana de Cirugía, Bogotá, D.C., Colombia

\section{Resumen}

Introducción. Hemos ingresado en una nueva era en la forma de comunicación y en la difusión de los contenidos que se generan en las diferentes reuniones y congresos científicos de Medicina y Cirugía.

Objetivo. Analizar los resultados de la difusión en Twitter ${ }^{\mathrm{TM}}$ del XLIV Congreso Nacional "Avances en Cirugía" realizado en Cartagena, Colombia, del I4 al I7 de agosto de 2018.

Método. Se recopilaron los datos obtenidos por la pagina Symplur.com en el XLIV Congreso Nacional "Avances en Cirugía" con la etiqueta (hashtag) \#CirugíaColombiana.

Resultados. Se generaron 76I tuits (tweets) durante todo el tiempo que duró el congreso, con I78 participantes activos en Twitter ${ }^{\mathrm{TM}}$, los cuales representan el Io, $8 \%$ del total de asistentes al congreso, con una relación de nueve asistentes por cada participante en Twitter ${ }^{\mathrm{TM}}$. En cuanto a la media de tuits por usuario, fue de cuatro y la cantidad de impresiones fue de I' 250.000. A medida que el congreso fue avanzando, aumentó el número de tuits, hasta el más alto, un total de 230, el 16 de agosto.

Discusión. El uso de twitter como herramienta de difusión por los cirujanos colombianos fue similar al publicado en otras series; sin embargo, son mediciones desde 20I3, en las cuales la participación y actividad han aumentado progresivamente. La canalización de los contenidos por medio de una etiqueta común permite la interacción asincrónica de todos los participantes, rompiendo las barreras de comunicación entre los mismos.

Palabras clave: cirugía general; congresos; redes de comunicación de computadores; red social; twitter.

Fecha de recibido: 16/05/2019 - Fecha de aceptación: 17/06/2019

Correspondencia: Diego Sierra, MD. Departamento de Cirugía, Universidad de La Sabana, Campus Puente del Común, Km 7, Autopista Norte de Bogotá, Chía, Colombia. Teléfono: 861-5555, extensión 23014

Correo electrónico: diego.sierra@unisabana.edu.co

Citar como: Sierra D, Sánchez EF, Sierra S, Márquez AC, Torregrosa L. La incursión del Congreso Nacional de Cirugía de Colombia en la red social Twitter'TM. Rev Colomb Cir. 2019;34:229-33. https://doi.org/10.30944/20117582.435

Este es un artículo de acceso abierto bajo una Licencia Creative Commons - BY-NC-ND https://creativecommons.org/licenses/by-nc-nd/4.0/deed.es 


\begin{abstract}
Introduction: We have entered a new era in the form of communication and the dissemination of the contents generated in the different meetings and scientific conferences of medicine and surgery

Objective: Analyze the results of the Twitter ${ }^{\circledR}$ broadcast of the 44th National Congress "Advances in Surgery" held in Cartagena - Colombia from August I4 to I7, 2018.

Methods: The data obtained by Symplur.com page was compiled in the 44th National Congress "Advances in Surgery" held in Cartagena - Colombia from August I4 to 17, 20I8, labeled with the Hashtag \#Colombian Surgery.

Results: 76I Tweets were generated during the whole time the conference lasted, with 178 active participants on Twitter which represent $10.8 \%$ of the total of attendees to the congress, with a ratio of 9 attendees for each participant on Twitter. As for the average of Tweets per user was 4 and the number of impressions was I'250,000. As the conference progressed there was more activity in the number of Tweets being the highest point on August I6 with a total of 230 Tweets.

Discussion: The use of Twitter as a dissemination tool by Colombian surgeons was similar to that published in other series, however, they are measurements since 2013, in which we have seen how participation and activity increases progressively. The channeling of the contents through a common Hashtag allows the asynchronous interaction of all the participants breaking communication barriers between them.
\end{abstract}

Key words: general surgery; congresses; computer communication networks; social networking; twitter.

\section{Introducción}

Es innegable que la era digital ha abierto todo un campo novedoso de formas de comunicación y difusión de contenidos para la Medicina, que no se restringe a la comunidad médica y las tradicionales publicaciones científicas, sino que abarca los terrenos de la investigación, los estudios clínicos, la educación profesional, los pacientes, las familias y el público en general, y genera nuevos espacios para el intercambio y la discusión entre colegas sin las barreras de tiempo, lugar y espacio. Además, la difusión de los contenidos generados en la práctica clínica cotidiana y en los espacios académicos que propician las diferentes reuniones y congresos de Medicina y Cirugía, ha enriquecido este ambiente virtual facilitando el acceso global y a bajo costo, a información científica actualizada y de calidad.

Lo anterior ha generado que herramientas como Twitter $^{\mathrm{TM}}$ sean cada vez más utilizadas, tanto por individuos como por entidades y organizaciones médicas. Los médicos clínicos e investigadores las emplean como un medio para compartir y transmitir sus ideas y proyectos de investigación, y propiciar la discusión académica en su campo de interés de una forma asincrónica, accesibe y sin barreras geográficas ${ }^{\mathrm{I}}$. Para las sociedades y revistas científicas que constituyen sus órganos oficiales de difusión, (por ejemplo, @JAmCollSurg, @BJSurgery, @JAMASurgery, @Cirugiaespanola y @ascolcirugia), las redes constituyen también medios de difusión de gran acogida e impacto, tanto para nativos como para inmigrantes digitales ${ }^{2}$.

Twitter ${ }^{\mathrm{TM}}$, como red social, funciona mediante la conexión de usuarios que, en sus cuentas creadas previamente, redactan textos de máximo I40 caracteres conocidos como tuits (tweets) sobre un tema particular o un contenido específico, a los cuales se pueden añadir imágenes y videos para compartirlos en tiempo real, lo cual genera una comunicación asincrónica con los demás usuarios de esta red. Estos tuits se pueden organizar según el tópico o tema de interés, el cual se identifica rápidamente mediante una etiqueta (hashtag) precedida del signo numeral (\#) 3 .

Se han comunicado diferentes experiencias previas en congresos médicos y científicos alrededor del mundo que usan redes sociales como Twitter $^{\mathrm{TM}}$ para difundir sus contenidos y lograr 
la conectividad entre sus participantes necesaria en la era digital.

El objetivo de este estudio fue analizar los resultados de la implementación del uso de la red social Twitter ${ }^{\mathrm{TM}}$, en el Congreso Nacional "Avances en Cirugía" realizado por la Asociación Colombiana de Cirugía en Cartagena, Colombia, del I4 al I7 de agosto de 2018.

\section{Metodología}

Se recopilaron los datos obtenidos en el Congreso, promovido por el Comité de Comunicaciones de la Asociación Colombiana de Cirugía desde la cuenta oficial de la Asociación @ascolcirugia con la etiqueta \#CirugíaColombiana.

La fuente de donde se obtuvieron los datos fue la página web Symplur.com, la cual permitió obtener información detallada de la actividad en Twitter $^{\mathrm{TM}}$ durante el periodo de tiempo establecido y, además, hacer seguimiento de todos los tuits emitidos que utilizaron la etiqueta \#CirugíaColombiana durante el Congreso. Además, esta herramienta permite identificar los usuarios que han tenido mayor actividad y que han generado más difusión de los contenidos durante el evento, estableciendo categorías según la cantidad de menciones y el número de tuits.

Las variables evaluadas fueron la cantidad de tuits que se emitieron durante las fechas de duración del congreso, el número de asistentes al congreso, el número de usuarios de Twitter que utilizaron la etiqueta, la media de tuits emitidos por cada participante y sus impresiones (número de veces que se ha visto o se ha interactuado con un tuit).

\section{Resultados}

$\mathrm{Al}$ analizar los resultados, se encontró que se generaron 76I tuits durante el tiempo que duró el Congreso, el cual contó con I.64I asistentes, de los cuales I78 (Io,8 \%) participaron activamente en Twitter; la relación fue de nueve asistentes por cada participante en Twitter, dato similar, o en algunos casos superior, a los de otras series publicadas previamente ${ }^{3,4}$. La media de tuits por usuario fue de 4 y la cantidad de impresiones fue de I' 250.000 .
A medida que el Congreso fue avanzando, aumentó el número de tuits hasta alcanzar el más alto, un total de 230, el I6 de agosto (figura I).

Las categorías obtenidas en la página Symplur.com durante el tiempo que duró el congreso, se agruparon por participantes, menciones $\mathrm{y}$ número de tuits (figura 2).

\section{Discusión}

En el periodo previo y durante el XLIV Congreso Nacional "Avances en Cirugía", realizado en Cartagena del I4 al I7 de agosto de 20I8, se diseñó una estrategia de medios orientada a las nuevas generaciones, considerando que las estrategias tradicionales -como las cartas impresas y el correo electrónico- han perdido progresivamente su utilidad pues, últimamente, son acogidas solo por los grupos etarios ajenos al entorno de las redes sociales y la mensajería instantánea.

Llevar a cabo una promoción activa a través de redes sociales, grupos profesionales de mensajería instantánea (Whatsapp ${ }^{\mathrm{TM}}$ ) y la página web de la Asociación Colombiana de Cirugía, permitió al Comité de Comunicaciones llegar a los cirujanos más jóvenes, estudiantes y residentes, popularizando la etiqueta \#CirugiaColombiana previamente inexistente en redes y que progresivamente se fue difundiendo entre los grupos académicos y servicios quirúrgicos en todo el país, para compartir y difundir contenidos de interés común, con un impacto muy favorable en la percepción general de "mayor inclusión" de las nuevas generaciones en las actividades de la Asociación.

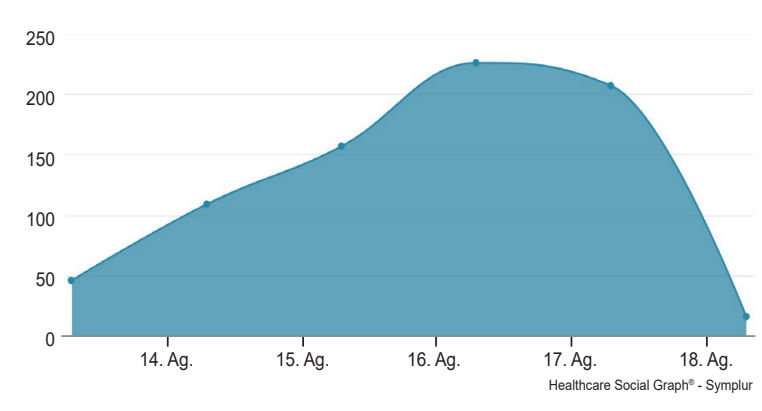

Figura 1. Actividad de Twitter en \#CirugiaColombiana 


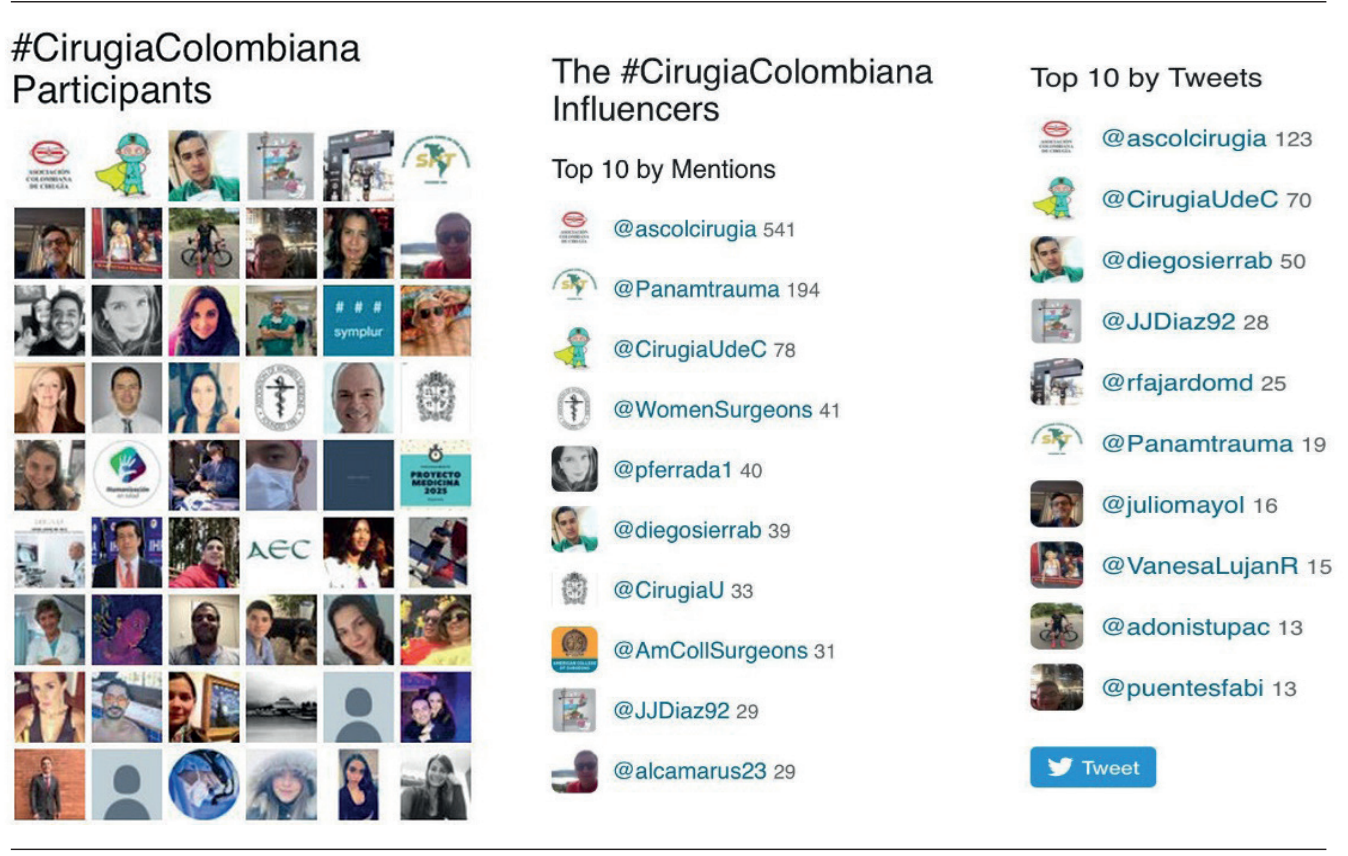

Figura 2. Ranking por categoría según participantes, menciones y número de tuits

Esta etiqueta también permitió la interacción entre los grupos quirúrgicos de diferentes regiones de Colombia, la participación de sus residentes en competencias fotográficas y la difusión de galardones y concursos. Al respecto, es interesante anotar que se ha descrito cómo la canalización de contenidos a través de una etiqueta común permite la interacción asincrónica de todos los participantes o interesados, rompiendo las barreras de comunicación entre los mismos 5 .

Consideramos que la actividad en Twitter ${ }^{\mathrm{TM}}$ por parte de los participantes en el Congreso fue significativa, teniendo en cuenta que constituyó una primera experiencia exploratoria y existían cuestionamientos frente a los resultados de la innovación en este terreno, previamente poco explorado por el gremio quirúrgico en nuestro país. El contar con esta línea de base será un buen punto de partida que permitirá más adelante mediciones de actividad y participación en eventos académicos y, seguramente, llevará a nuevas propuestas para optimizar la utilización de las redes virtuales en beneficio de la academia y de la profesión.
El uso de esta herramienta de difusión por cirujanos colombianos fue similar al publicado en otras series sobre congresos internacionales ${ }^{4}$, teniendo en cuenta que se han reportado mediciones desde el año 20I3, con un incremento progresivo en la participación y la actividad en Twitter ${ }^{\mathrm{TM}}$.

El uso de Twitter ${ }^{\mathrm{TM}}$ en eventos médicos ha experimentado una rápida expansión en los últimos años y, en las diversas experiencias reportadas sobre reuniones científicas alrededor del mundo, se concluye que el manejo activo de los medios y de las redes sociales, especialmente Twitter $^{\mathrm{TM}}$, permiten la promoción de los eventos académicos y la difusión de información, y además, hacen posible la comunicación y el intercambio fluido de opiniones, así como la discusión de los contenidos de las conferencias a nivel local, regional y mundial.

Las cuentas en Twitter ${ }^{\mathrm{TM}}$ de las sociedades científicas, además de citar nuevos artículos y colaborar en su difusión, permiten atraer a los cirujanos jóvenes, residentes, internos y estudiantes interesados en la Cirugía, y agruparlos en torno a temas de interés mutuo, lo cual facilita 
el intercambio y la discusión de conocimientos de manera constante. De igual modo, permiten y mejoran la promoción de cursos, congresos, campañas y estudios clínicos. Es por esto que ya se considera una fuente de noticias válida y fiable en el ámbito médico y científico.

Por la experiencia de los vertiginosos cambios que han ocurrido en los últimos años en cuanto a la difusión de la información médica, se puede pronosticar cada vez mayor interacción directa entre los miembros de nuestra comunidad a través de las 'redes sociales' vigentes y muchas otras que aparecerán para facilitar novedosas formas de comunicación, interacción y difusión de contenidos y experiencias entre los miembros de la profesión y también entre los médicos y la sociedad.

\section{Referencias}

I. Cochran A, Kao LS, Gusani NJ, Suliburk JW, Nwomeh BC. Use of Twitter to document the 2013 Academic Surgical Congress. J Surg Res. 20I4;190:36-40. doi: IO.IOI6/j. jss.2014.02.029

2. Segura JJ, Morales R, Ramos JL, González-Argenté FJ, Mayol J. Uso de Twitter ${ }^{\circledR}$ y sus implicaciones en las reuniones y congresos de la Asociación Española de Cirujanos. Cirugía Española. 2018;96:352-6.

3. Chaudhry A, Glodé LM, Gillman M, Miller RS. Trends in Twitter use by physicians at the American Society of Clinical Oncology Annual Meeting, 2010 and 20II. J Oncol Pract. 20I2;8:173-8. doi: Io.I200/JOP.20II.000483

4. Wilkinson SE, Basto MY, Perovic G, Lawrentschuk N, Murphy DG. The social media revolution is changing the conference experience: Analytics and trends from eight international meetings. BJU Int. 2015;II5:839-46.

5. Logghe HJ, Selby LV, Boeck MA, Stamp NL, Chuen J, Jones C. The academic tweet: Twitter as a tool to advance academic surgery. J Surg Res. 2018;226:viii-xii. doi: I0.IoI6/j.jss.20I8.03.049 